BJD, CONCISE COMMUNICATION

Dopamine is a novel, direct inducer of catagen in human scalp hair

follicles

EA Langan, ${ }^{1,2}$ E Lisztes, ${ }^{3}$ T Bíró ${ }^{3}$ Funk W, ${ }^{4}$ CEM Griffiths, ${ }^{1}$ R Paus ${ }^{1,2}$.

${ }^{1}$ Dermatology Research Centre, The University of Manchester, Manchester Academic Health Science Centre,

Manchester, UK

${ }^{2}$ Experimental Dermatology, Universitätsklinikum Schleswig Holstein, Lübeck, Germany

${ }^{3}$ DE-MTA “Lendulet” Cellular Physiology Research Group, Department of Physiology, University of Debrecen, H-4032 Debrecen, Hungary

${ }^{4}$ Dr Clinic Dr. Kozlowski, Munich.

Running title: Dopamine promotes catagen

Word Count $\quad: 1123$ excluding abstract (MAX 1100)

Figures $\quad: 2($ MAX 2)

eSupplementary Figures $\quad: 3$

Abstract : 221 words (MAX 250)

References $\quad: 19($ MAX 15)

Funding : EAL is supported by a Medical Research Council (UK)

Clinical Research Training Fellowship.

Conflict of Interest $\quad$ : EAL and RP have filed a patent on the hair growth inhibitory

effects of dopamine

Corresponding Author $\quad$ : Ralf Paus 
What's already known about this topic? (70 words)

- There are reports, almost exclusively in females, associating treatment with L-Dopa or dopaminergic agoinsts with diffuse hair loss.

What does this study add?

- Dopamine is a direct inhibitor of female human hair growth, at least in vitro, via the promotion of catagen induction.

- Preliminary evidence that dopamine 1 receptor transcripts are present in human hair follicles and skin, suggesting that dopamine receptor agonists warrant exploration as novel inhibitors of unwanted human hair growth (hirsutism, hypertrichosis). 
Background: Although there are clinical reports of hair loss associated with levodopa and dopaminergic agonists, it is unclear whether dopamine exerts any direct effects on the human hair follicle (HF).

Objectives: Given the widespread use of dopamine agonists and antagonists in clinical medicine, we sought to determine whether dopamine exerts direct effects on human HF growth and pigmentation in vitro and whether human HFs express dopamine receptors (DR).

Methods: Microdissected human scalp HFs from three female patients (age 47-68 years) were treated in serum-free organ culture for 7 days with dopamine (10-1000nM), and its effects on hair shaft production, HF cycling (i.e. anagen-catagen transition), hair matrix keratinocyte proliferation/apoptosis, and HF pigmentation were measured by quantitative (immuno-) histomorphometry.

Results: Dopamine had no consistent effect on hair shaft production, but did promote HF regression (catagen). It was also associated with significantly reduced proliferation of HF matrix keratinocytes $(\mathrm{p}<0.01)$ and reduced intrafollicular melanin production. Dopamine receptor (DR1) transcripts were identified in HFs and skin.

Conclusions: These data provide evidence that dopamine is a direct inhibitor of human hair growth, via the promotion of catagen induction. This may offer a rational explanation for the induction of telogen effluvium in some female patients treated with dopaminergic agonists such as bromocriptine. Moreover, dopaminergic agonists deserve further exploration as novel inhibitors of unwanted human hair growth (hirsutism, hypertrichosis). 


\section{Introduction}

Treatment with levodopa or dopaminergic agonists has been associated with the development of diffuse hair loss, predominantly in females. In most of the reported cases this hair loss was reversible after cessation of medication, although in some, it persisted ${ }^{1,2}$. More recently, telogen effluvium has also been reported in association with the dopamine agonist pramipexole in a woman with Parkinsons's disease ${ }^{3}$ leading to speculation that hair loss may be overlooked in such patients ${ }^{4}$.

However, it is unknown whether dopamine and other dopamine receptor agonists directly impact human hair growth, rather than modulating other hormones that regulate hair growth, and whether human scalp hair follicles (HFs) express dopamine receptors (DRs). Dopamine acts via G-protein coupled DRs, of which five types have been identified. DR are classified on the basis of their effect on adenylate cyclase, with D1R and D5R being stimulatory and D2R, D3R and D4R inhibitory ${ }^{5}$. Peripheral dopamine production is thought to arise from neurons, the adrenal medulla and neuroendocrine cells ${ }^{6}$ Interestingly, dopamine agonists can exert anti-proliferative effects on cultured human dermal fibroblasts ${ }^{7}$, and dopamine may regulate angiogenesis during cutaneous wound healing ${ }^{8}$. Human skin possesses the essential enzymes not only for dopamine metabolism, but also for dopamine synthesis, namely, dihydroxyphenylalanine (DOPA) decarboxylase and dopamine $\beta$ hydroxylase ${ }^{9}$; human HF transcribe the DOPA decarboxylase gene ${ }^{10}$.

Given the widespread use of both dopamine agonists and antagonists in clinical practice, we therefore asked whether dopamine exerts direct effects on human HF growth and pigmentation in vitro and whether they express DRs. This was studied in serum-free organ culture of normal female scalp HFs. 


\section{Materials and Methods}

Microdissected anagen VI HFs were obtained from redundant scalp skin, as a by-product of cosmetic surgery, with ethical approval (University of Lübeck, Reference 06-109), adherence to the Helsinki Declaration principles, and written informed consent. Microdissected HFs were cultured in supplemented serum-free culture medium protocol for 7 days as described ${ }^{11}$ with the addition of dopamine (protected from light; Sigma-Aldrich, Taufkirchen) 10nM $1000 \mathrm{nM}$ or culture medium only. These concentrations were selected on the basis of previous studies, with the addition of a higher dopamine concentration $(1000 \mathrm{nM})^{5}$. Hair shaft elongation was measured as percentage elongation from baseline, and hair cycle stage and hair cycle score determined morphometrically as described ${ }^{12}$. HFs were embedded in Cryomatrix (Thermo Shandon, Cheshire, UK), snap frozen in liquid nitrogen, cryosectioned $(6 \mu \mathrm{m})$ and Ki/TUNEL ${ }^{13}$, Masson-Fontana, tyrosinase activity in situ and Gp100 immunoreactivity (IR) ${ }^{14}$ were performed as previously reported. Statistical analysis was performed as detailed in Fig.1. "Real time" PCR for was performed as described in (Supplementary Fig. 3b).

\section{Results}

Dopamine 1000nM increased the percentage of HFs in the catagen phase of the hair cycle, which was consistent with a significant increase in the hair cycle score ( $p=0.03$; Fig. 1a, b). Hair cycle score, calculated as previously described, accurately indicates hair cycle stage ${ }^{15}$. This was independently confirmed by assessing HF pigmentation, since catagen development switches off melanin production. Masson-Fontana quantitative histomorphometry showed a significant reduction in melanin staining $(\mathrm{p}=0.04)$ (Fig. 1c-e), consistent with premature anagen termination by dopamine treatment. Tyrosinase activity in situ and Gp100 IR were not significantly affected by dopamine 1000nM (Supplementary Fig. 1a and b). When pigmentary parameters were compared only between test and control HFs that were all in 
anagen VI, there was no hair cycle-independent effect of dopamine on intrafollicular pigmentation (Supplementary Fig. 2a-k, Supplementary Fig. 3.)

Next, the percentage of Ki67 positive cells in the hair matrix below Auber's line, the widest part of the dermal papilla, was assessed in anagen VI HFs. Dopamine 100nM and 10nM significantly reduced the percentage of Ki67 positive cells when compared to the control group (Fig 2a-f), in line with the catagen promoting effects of dopamine noted above. However, there was no significant increase in the number of apoptotic (TUNEL+) cells at 100 or 10nM. Dopamine had no consistent effect on shaft elongation in the HF from 4 different patients analyzed, at the concentrations tested (Supplementary Fig. 1c and d). However, there was a (non-significant) trend towards decreased shaft production in the $10 \mathrm{nM}$ treatment group.

Since these findings showed that dopamine can directly inhibit the growth of isolated human HF in serum-free organ culture, this suggested that human scalp HFs also express DRs. Indeed, DR1 transcription was demonstrated in both HFs and skin. That was indeed the case was confirmed by "real time" PCR (Supplementary Fig. 3b).

\section{Discussion}

This study provides the first direct evidence that dopamine treatment negatively affects human hair growth, namely that it promotes HF regression (catagen) in female anagen VI HFs in organ culture, i.e. in the absence of neural or vascular cues. This is entirely consistent with the in vivo case reports of hair loss in patients being treated with dopaminergic agonists ${ }^{1,3,4,16,17}$. Moreover, this study demonstrates that dopamine reduces HF matrix keratinocyte proliferation. 
Whilst the precise mechanism(s) by which dopamine induced premature catagen development in female human anagen HFs remains to be elucidated, the intrafollicular presence of DR transcripts suggests a DR-mediated mechanism (Supplementary Fig 3b). It has already been shown that murine skin expresses DRs ${ }^{18}$, with DR2 localised to the basal layer of the epidermis. DR2 agonist treatment resulted in reduced epidermal hyperplasia in response to barrier disruption. In cultured human epidermal keratinocytes, treatment with the dopaminergic agonist bromocriptine also reduced proliferation ${ }^{18}$. Our finding that dopamine is a catagen promoter and can inhibit human hair matrix keratinocyte proliferation in situ is in line with these studies.

Given the evidence that prolactin treatment can stimulate the growth of female frontotemporal HFs in vitro ${ }^{19}$, we speculate that dopamine could have reduced intra-follicular prolactin (PRL) levels, just as it reduces PRL secretion from the pituitary gland, thus exerting the observed hair growth effects. This hypothesis needs to be addressed in future research. However, it should be borne in mind that in the clinical context levodopa is often administered with a peripheral DOPA decarboxylase inhibitor, thus the amount of Dopamine reaching, or being converted in the HF is unclear. Future studies should also address the effect of levodopa on the HF.

Clinically, these data shed new light on the neuroendocrinology of human hair growth and provide encouraging preclinical evidence that dopamine and DR agonists may be useful as novel anti-hirsutism agents, whilst raising the possibility that DR antagonists may exert novel hair growth promoting effects. Of course, this must be confirmed in larger numbers of HFs in vitro before this can be tested in vivo. Further work is also required to investigate the effect of dopamine on hair growth in males, and to determine the expression and regulation of the 
range of intrafollicular DRs (1-5) which may uncover much needed new targets for the management of hair growth disorders.

\section{Figure Legends}

\section{Figure 1. Dopamine significantly increases hair cycle score, and decreases follicular} pigmentation in a hair cycle dependent manner. Dopamine treatment resulted in an increased percentage of HFs in catagen (a) and a significant increase in Hair cycle score $(p=0.03), 27-37$ HFs per group from 3 females aged 47-68 (b). When melanin content in all HFs was measured, 1000nM dopamine treatment significantly reduced follicular pigmentation (c). $\mathrm{p}=0.004,27-38 \mathrm{HFs}$ per group from 3 females aged 47-68. Melanin content was reduced between control (d) and 1000nM dopamine treated HFs (e). For statistical analyses all data was checked for normal distribution using the D'Agostino and Pearson normality test. If normally distributed, students t-test or one way ANOVA, with Bonferroni's post hoc test, were used where appropriate. If the data were not normally distributed, or the number was too small to confirm this, groups were compared using Mann-Whitney U-test or Kruskal-Wallis test, with Dunn's multiple comparison post hoc test, where appropriate. Statistical analysis was performed using GraphPad Prism, Version 5, San Diego California, USA. Statistical significance was considered as $\mathrm{p}<0.05$ and results were expressed as the mean $+/$ - standard error of the mean (SEM). To combine the results from the independent quantitative (immuno-) histomorphometry experiments, the results from the treatment groups were normalized to the control group (set at 100) and the results pooled. Therefore the control group always has a mean of $100+/$ - the SEM. Reference areas for the measurement of tyrosinase IR and melanin staining and are shown in 1f-g. 


\section{Figure 2. Dopamine significantly decreases matrix keratinocyte in female human hair}

follicles. Comparing anagen VI HFs at the end of culture, Dopamine 10-100nM significantly decreased the percentage of Ki67 positive cells below Auber's line in two female patients aged 47-64, 13-20 HFs per group (a). There was no difference in TUNEL positive cell number in the equivalent area (b). Ki67 positive cells in the control group (c) and dopamine treatment groups 1000nM (d), $100 \mathrm{nM}(\mathrm{e})$ and 10nM (f).

\section{Supplementary Figure 1. Dopamine does not significantly reduce Gp100 immunoreactivity,} tyrosinase IR or hair follicle elongation. Gp100 (22-23 HFs/group, 3 females aged 47-68) (a) and tyrosinase IR (9-13 HFs/group, 2 females aged 64-68) (b) was reduced when all HFs were measured, the decrease was not statistically significant. Dopamine $1000 \mathrm{nM}$ had no significant effect on hair follicle (HF) elongation in vitro (c). 54 HFs were treated in both groups from 3 females aged 47-68 and results pooled. Lower concentrations of DA (10-100nM) exerted no consistent effects of HF elongation (d). 53-54 HFs in each group from 3 female patients aged 47, 52 and 64.

\section{Supplementary Figure 2. Dopamine exerts no hair cycle independent effects on several} parameters of follicular pigmentation. To determine whether dopamine treatment had any effect on HF pigmentation, Masson Fontana staining was performed. There was no significant difference in pigmentation at any concentration of dopamine tested, when only anagen VI test and control HFs were compared (a). 20-23 HFs per group from three 3 females aged 47-68. Melanin pigmentation in the control group (b), and Dopamine treatment groups 1000nM (c), 100nM (d) and 10nM (e) was not significantly different. Consistent with these results, tyrosinase immunoreactivity (IR) (f) was not changed. Control group HF (g) and 1000nM dopamine treated HF (h). 2 female patients, 64-68 years, 9-12 HFs per group. Moreover. 
Gp100 IR (f) was not changed (i). 3 female patients aged 47-68, 15-18 HFs per group. Control

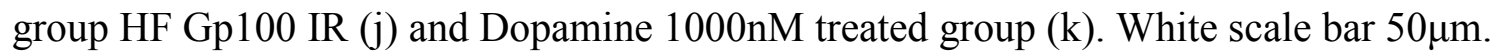

\section{Supplementary Figure 3. Dopamine receptor 1 transcripts are present in human skin and hair} follicles and persist in organ culture (a) The skin contains the key enzymes required for pigmentation, several of which may be targets of dopamine. Although tyrosinase IR was not significantly influenced by dopamine, the mechanism(s) regulating the hair cycle dependent effects of dopamine on follicular pigmentation need to be further investigated. (b) Dopamine 1 receptor (DR1) expression was identified using quantitative "real-time" PCR. This was

performed as described previously ${ }^{13}$ using TaqMan primer for DRD1 Hs00265245_s1. The blue columns represent DR1 expression in skin from the same subject (42 year old female) at different point in the culture. Culture was with supplemented Williams E media. The red column represents DR1 expression at day 7 in skin from a 63 year old female. Purple column represents DR1 expression in fresh HFs (57 year old female), and DR1 expression in HFs after 48 hours in culture is shown in the yellow column (66 year old female.)

\section{Acknowledgements}

The technical assistance of Ms. G. Scheel, C Kremling and A. Winter-Keil is gratefully acknowledged. 


\section{References}

1 Grauer MT, Sieb JP. Alopecia induced by dopamine agonists. Neurology 2002; 59: 2012.

$2 \quad$ Marshall A, Williams MJ. Alopecia and levodopa. Br Med J 1971; 2: 47.

3 Katz KA, Cotsarelis G, Gupta R et al. Telogen effluvium associated with the dopamine agonist pramipexole in a 55-year-old woman with Parkinson's disease. J Am Acad Dermatol 2006; 55: S103-4.

4 Miwa H, Kondo T. Hair loss induced by dopamine agonist: case report and review of the literature. Parkinsonism Relat Disord 2003; 10: 51-2.

5 Borcherding DC, Hugo ER, Idelman G et al. Dopamine receptors in human adipocytes: expression and functions. PLoS One 2011; 6: e25537.

6 Rubi B, Maechler P. Minireview: new roles for peripheral dopamine on metabolic control and tumor growth: let's seek the balance. Endocrinology 2010; 151: 5570-81.

7 Laengle UW, Markstein R, Pralet D et al. Effects of latanoprost and GLC756, a novel dopamine D2 agonist and D1 antagonist, on cultured normal human dermal fibroblasts. Eur J Ophthalmol 2006; 16: 67-72.

8 Shome S, Rana T, Ganguly S et al. Dopamine regulates angiogenesis in normal dermal wound tissues. PLoS One 2011; 6: e25215.

9 Ramchand CN, Clark AE, Ramchand R et al. Cultured human keratinocytes as a model for studying the dopamine metabolism in schizophrenia. Med Hypotheses 1995; 44: 53-7.

10 Chang YT, Hyland K, Mues G et al. Human hair follicles as a peripheral source of tyrosine hydroxylase and aromatic L-amino acid decarboxylase mRNA. Neurosci Lett 1997; 222: 210-2. 
11 Lu Z, Hasse S, Bodo E et al. Towards the development of a simplified long-term organ culture method for human scalp skin and its appendages under serum-free conditions. Exp Dermatol 2007; 16: 37-44.

12 Kloepper JE, Sugawara K, Al-Nuaimi Y et al. Methods in hair research: how to objectively distinguish between anagen and catagen in human hair follicle organ culture. Exp Dermatol 2010; 19: 305-12.

13 Ramot Y, Biro T, Tiede S et al. Prolactin--a novel neuroendocrine regulator of human keratin expression in situ. Faseb $J$ 2010; 24: 1768-79.

14 Gaspar E, Nguyen-Thi KT, Hardenbicker C et al. Thyrotropin-releasing hormone selectively stimulates human hair follicle pigmentation. J Invest Dermatol 2011; 131: 2368-77.

15 Samuelov L, Sprecher E, Tsuruta D et al. P-Cadherin Regulates Human Hair Growth and Cycling via Canonical Wnt Signaling and Transforming Growth Factor-beta2. $J$ Invest Dermatol 2012.

16 Gautam M. Alopecia due to psychotropic medications. Ann Pharmacother 1999; 33: $631-7$

17 Tabamo RE, Di Rocco A. Alopecia induced by dopamine agonists. Neurology 2002; 58: $829-30$.

18 Fuziwara S, Suzuki A, Inoue K et al. Dopamine D2-like receptor agonists accelerate barrier repair and inhibit the epidermal hyperplasia induced by barrier disruption. $J$ Invest Dermatol 2005; 125: 783-9.

19 Langan EA, Ramot Y, Goffin V et al. Mind the (gender) gap: does prolactin exert gender and/or site-specific effects on the human hair follicle? J Invest Dermatol 2010; 130: $886-91$. 


\title{
Dopamine is a novel, direct inducer of catagen in human scalp hair follicles.
}

\author{
Langan EA, Lisztes E, Bíró T, \\ Funk W, Griffiths CE and Paus R
}


(a)

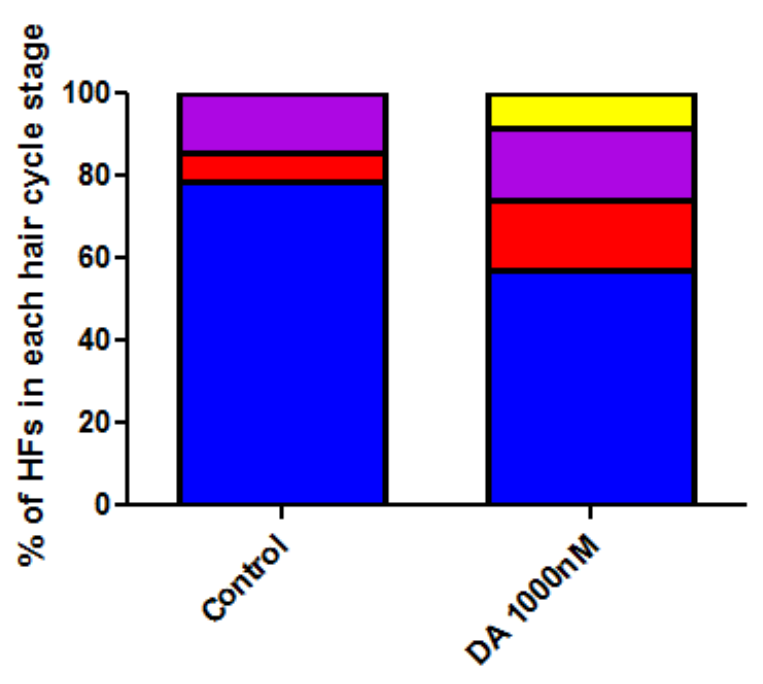

(c) Pigmentation: Masson Fontana Staining Intensity Hair cycle dependent

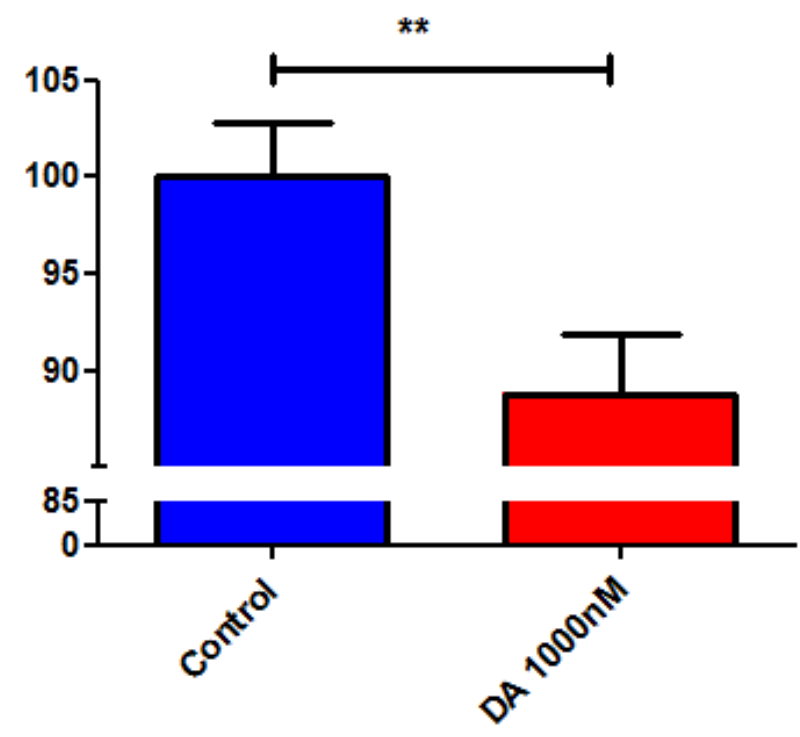

(b)
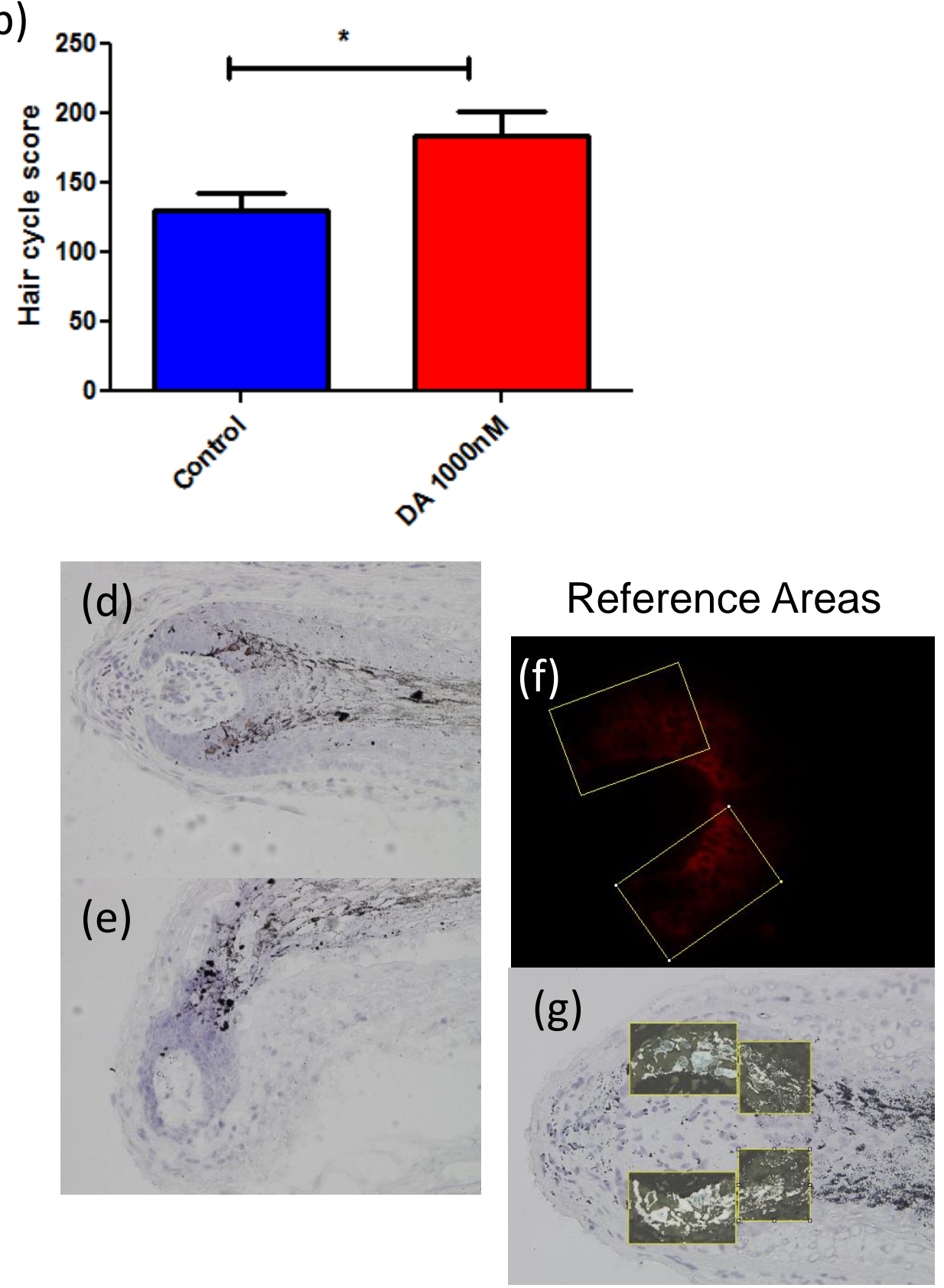

Figure 1. Dopamine significantly increases hair cycle score and decreases follicular pigmentation in a hair cycle dependent manner 
(a)

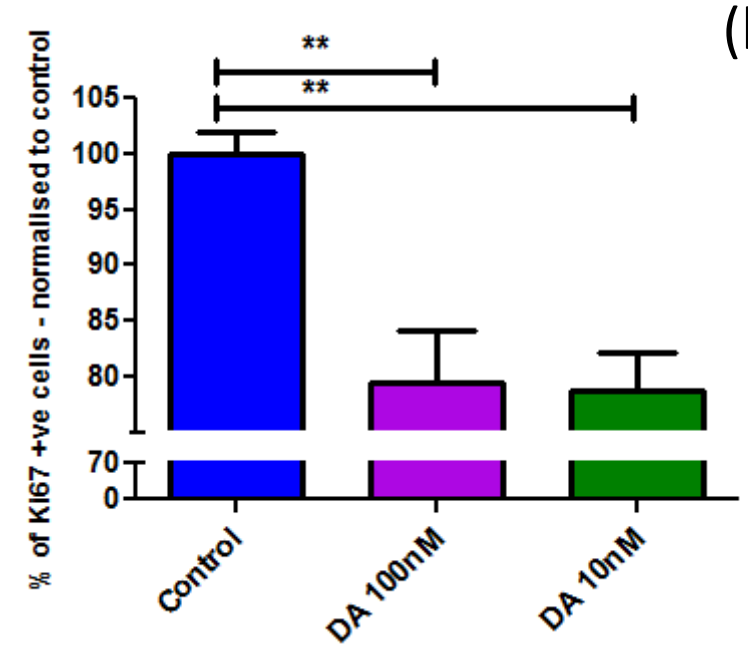

(b)

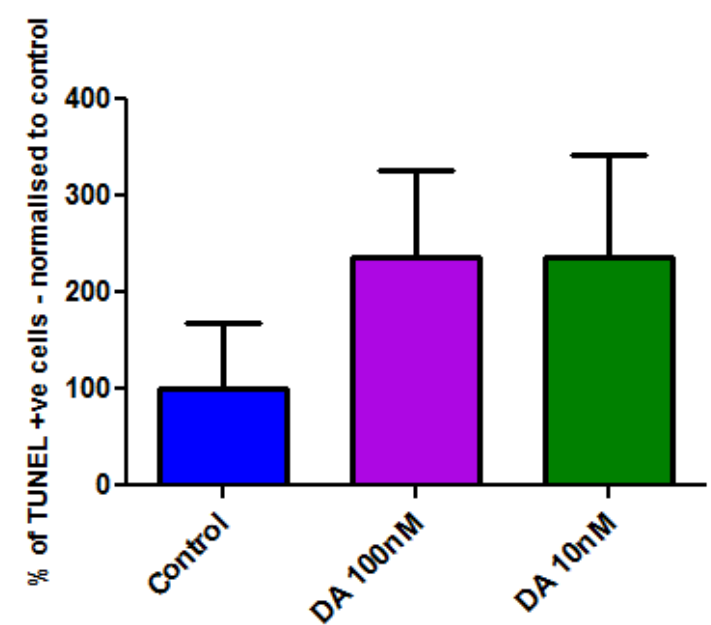

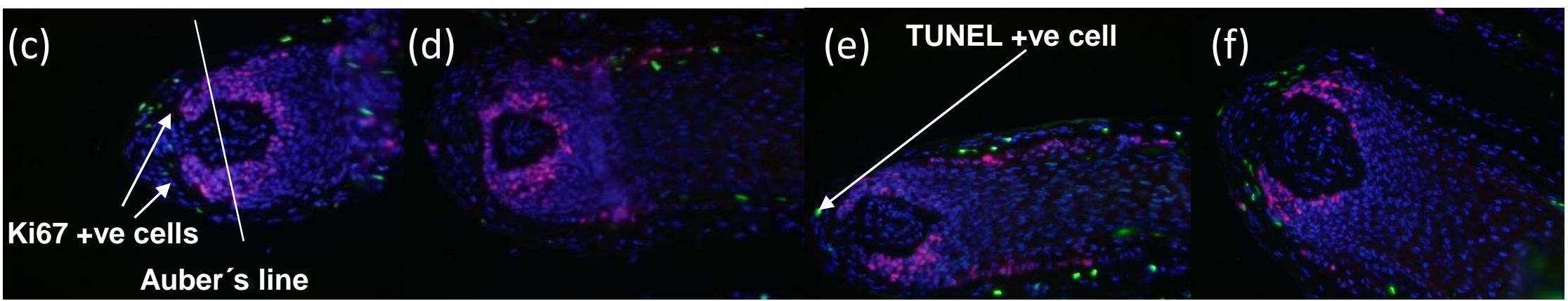

Figure 2. Dopamine significantly decreases matrix keratinocyte in female human hair follicles. 


\section{(a)}

8

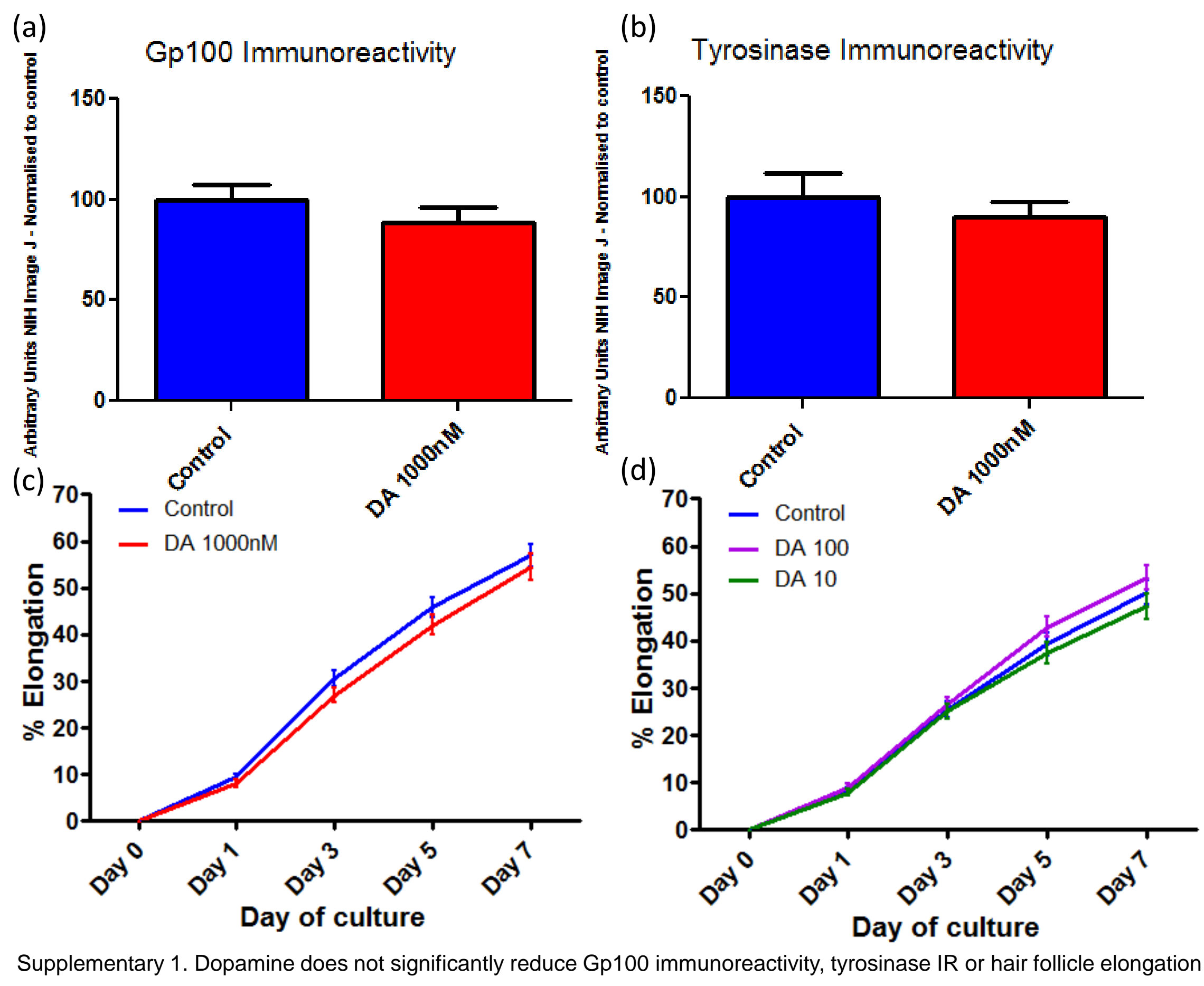

(b)

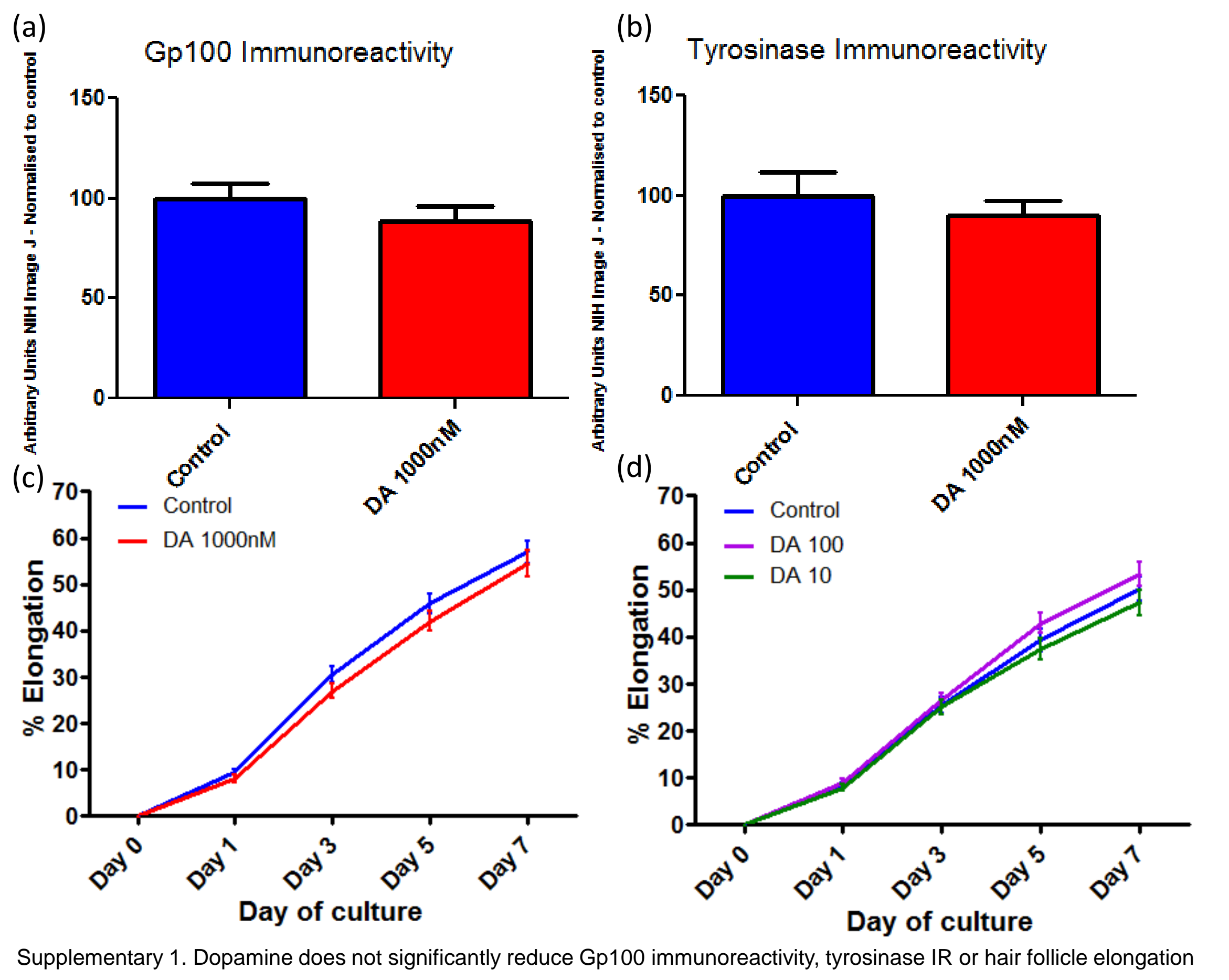

Supplementary 1. Dopamine does not significantly reduce Gp100 immunoreactivity, tyrosinase IR or hair follicle elongation

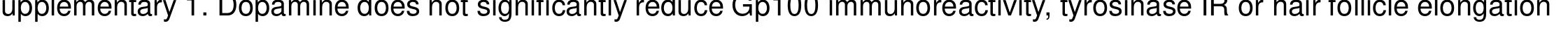

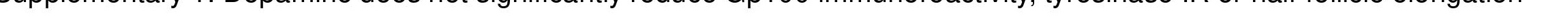

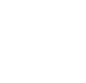

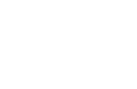

(201 
(a)

Pigmentation: Masson Fontana staining intensity

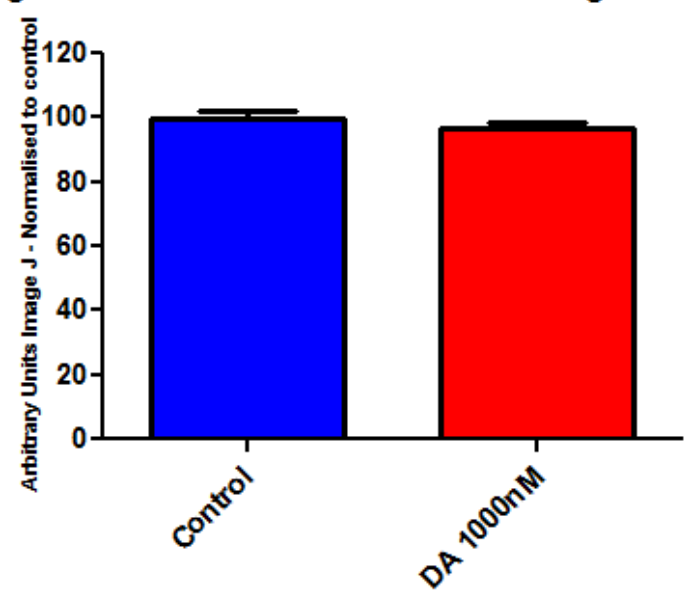

(b)

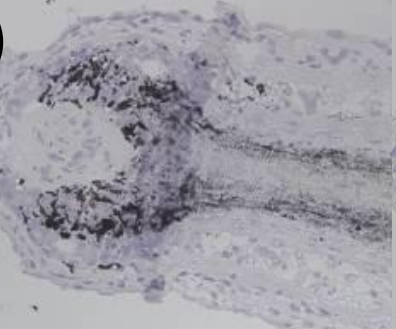

(c)

(d)

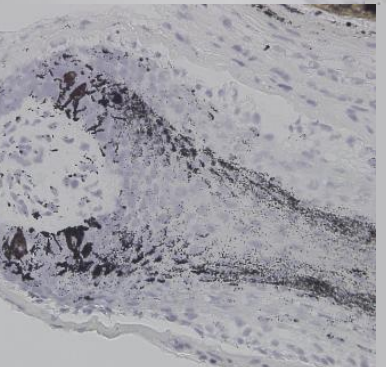

(e) (f)
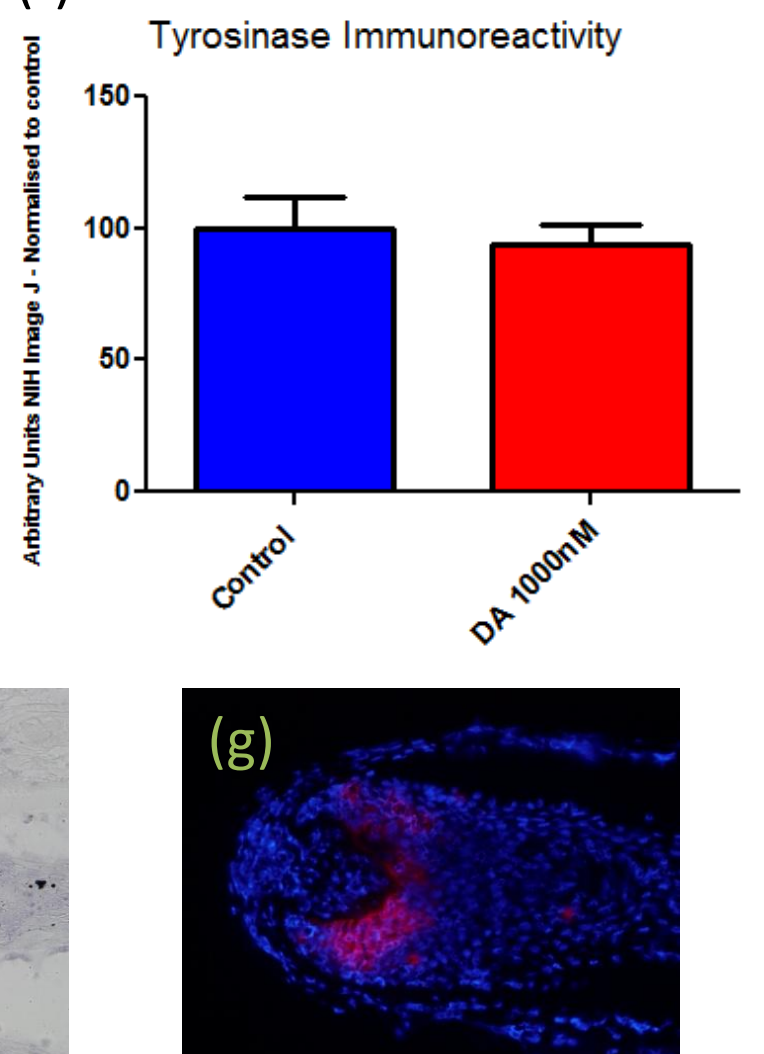

(h) (i)
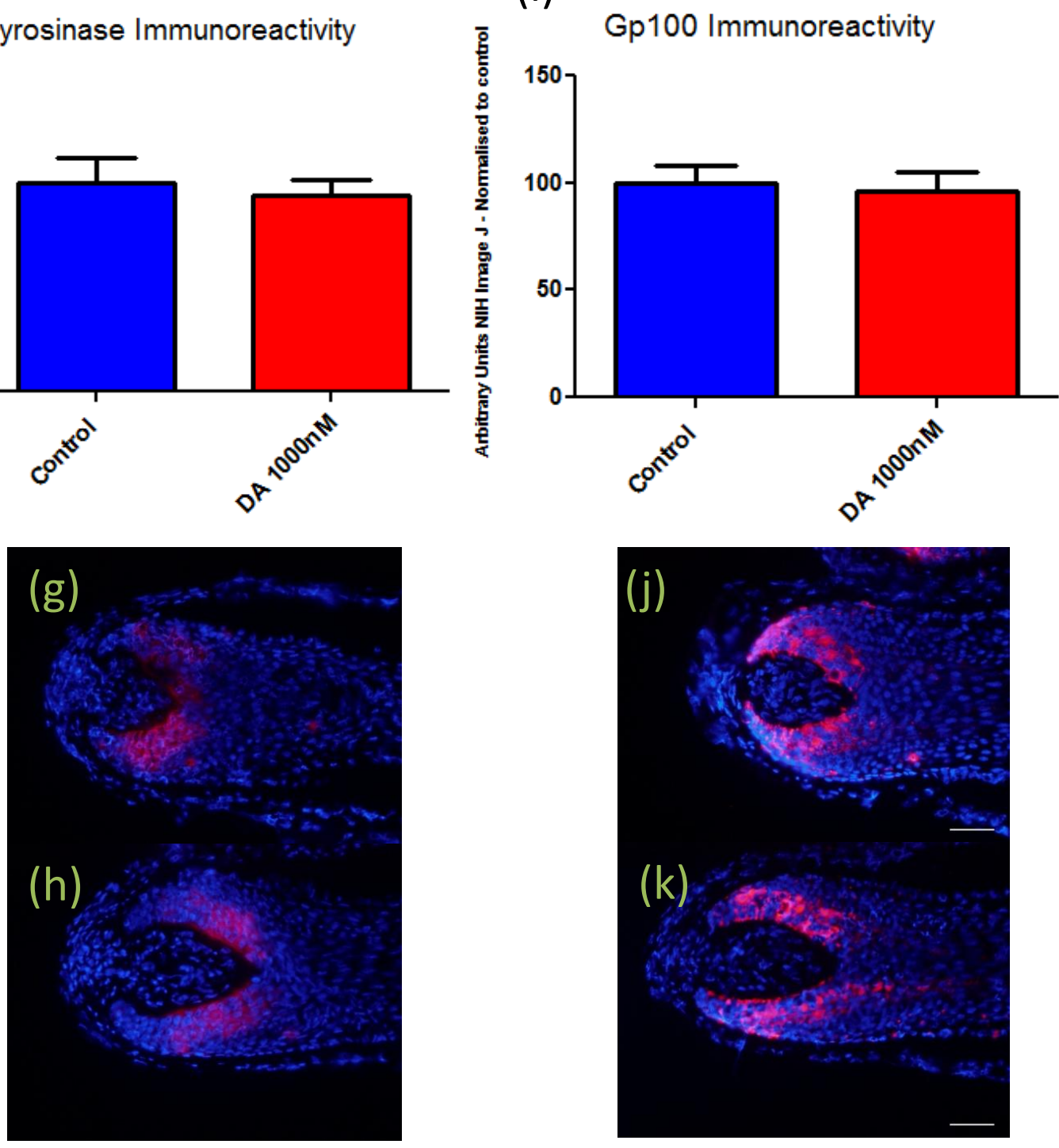

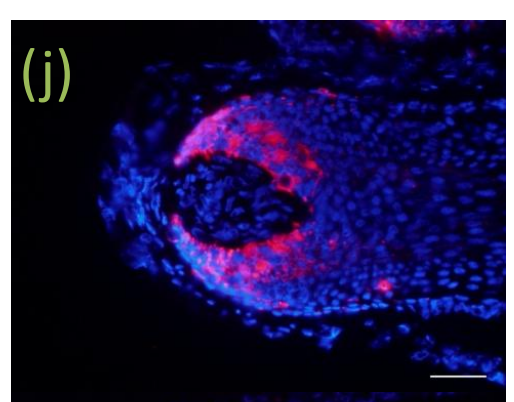

(k)

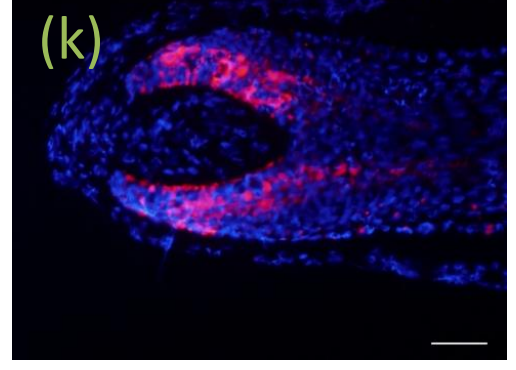

Supplementary 2. Dopamine exerts no hair cycle independent effects on several parameters of follicular pigmentation. 
(a) PHA hydroxylase
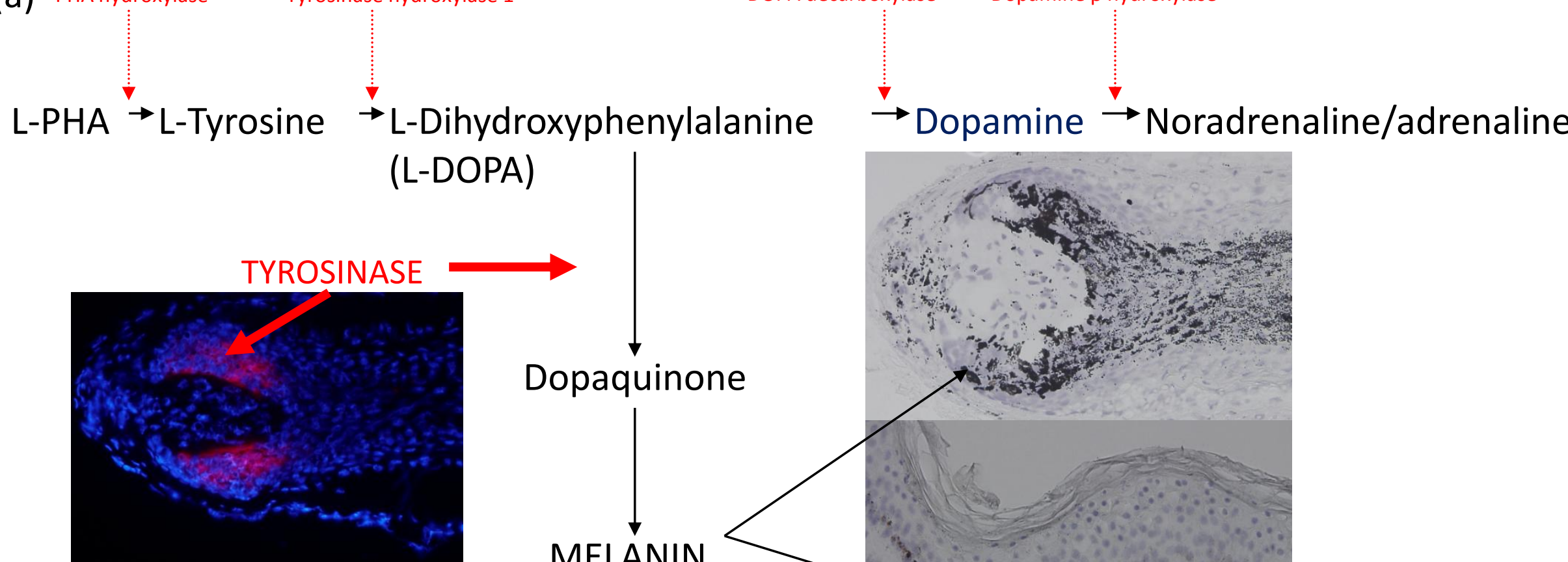

Red: Enzymes involved in the pigmentation pathway.

Abbreviation: PHA: Phenylalanine hydroxylase

(b)

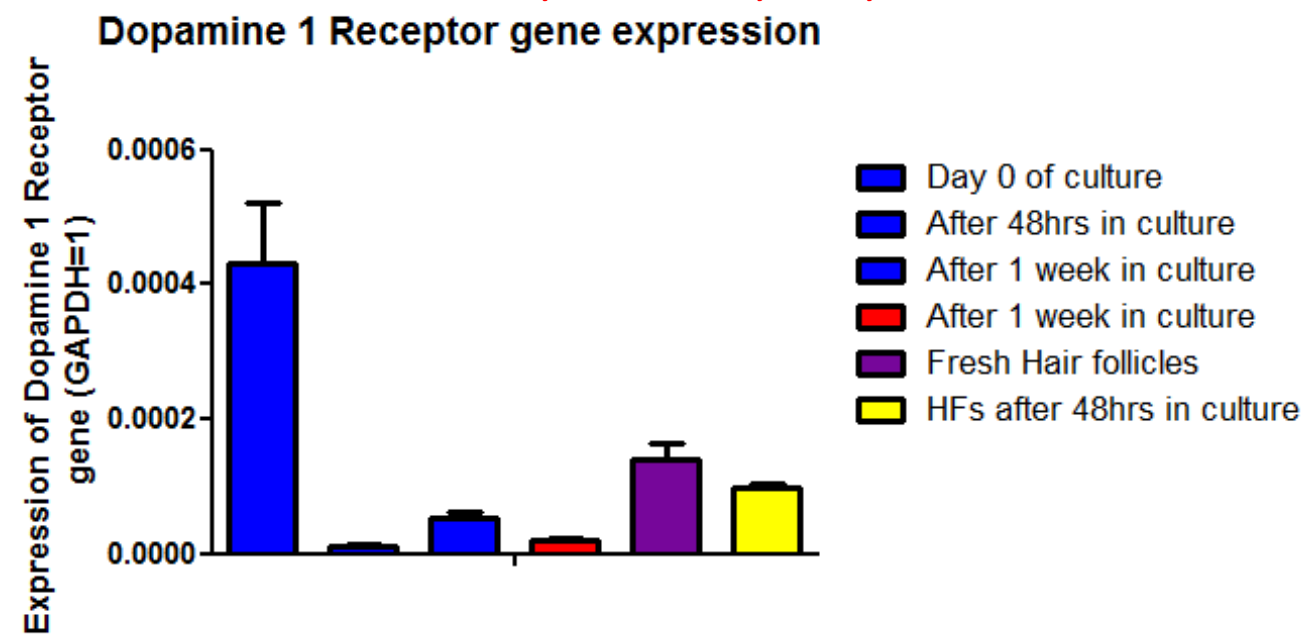

Supplementary 3. Dopamine receptor 1 transcripts are present in human skin and hair follicles and persist in organ culture 